\title{
A New Approach for Dynamic Time Quantum Allocation in Round Robin Process Scheduling Algorithm
}

\author{
Ritika Gakher, \\ Department of Computer Science, FMIT \\ Jamia Hamdard, New Delhi
}

\author{
Saman Rasool, \\ Department of Computer Science, FMIT \\ Jamia Hamdard, New Delhi
}

\begin{abstract}
Round-Robin (RR) is the vastly used process scheduling algorithm where, all processes are allocated some time shares in a circular order. If a process is able to complete its execution within this time quantum share, it is removed from the ready queue else it goes back at the end of the queue. Round-Robin scheduling is simple, gives fair allocation of CPU to the process and is starvation free. However there is few performance issues related to it. One of them is that even if there is a fractional amount of time left for a process to complete it execution, and its time share expires, it is preempted. Now this process has to wait unnecessarily to get its next chance to complete this fractional remaining execution. In this paper the proposal for the modification which will eradicate this performance issue of the conventional round robin algorithm is proposed thereby making it fairer.
\end{abstract}

\section{Keywords}

Time Quantum, Turnaround Time, Number of Cycles, Remaining Time, Number of Context switches, Wait time.

\section{INTRODUCTION}

An operating system may be defined as the interface between the user and the hardware.[1][2] Among numerous functionalities of the operating system, the most essential one is the job of process scheduling in which the operating system chooses which process will be executed first and in what manner.[3][4][6]

Benchmark of a good scheduling algorithm depends on the following criteria:

- All processes should get equal share at the central processing unit.

- $\quad$ CPU should be at work all the time i.e. it should not be idle.

- $\quad$ Response time should be minimized.

- Numbers of jobs executed per unit time should be maximized

There are several scheduling algorithms available some of them include:

\subsection{First Come First Serve (FCFS)}

In this algorithm, the process that reaches first at the CPU will be executed first. The CPU will be accessible to the process until the time the process completes its execution or performs an I/O operations.[1][10][11].

\subsection{Shortest Job First (SJF)}

In this algorithm the process that has the shortest burst time will be executed first by the CPU, then the process with the next higher burst time will be executed and so on. Here also the CPU will be available to the process until the time the process completes its execution or performs an I/O operation $[3][4][7][10]$

\subsection{Round Robin (RR)}

The viewpoint of this algorithm is similar to that of FCFS except the fact that the process will be executed by the CPU for a fixed time quantum. If the process gets completed within this period, it is removed from the ready queue otherwise it goes back at the end of the ready queue and waits for its next chance.[1][3][5]

\subsection{Priority Scheduling}

In this algorithm, the process with the highest priority is arranged first thereby executed first by the CPU. [6][7]

\subsection{Multilevel queue scheduling}

In this the ready queue is segregated into a number of different queues. The assignment of the processes to one of the queue permanently is usually based on properties of the processes such as memory size, process priority of type of process.[3][10][11]

\subsection{Multilevel feedback queue scheduling}

This algorithm is similar to the multilevel queue scheduling but it permits process to move between the queues.[9][10][11]

\subsection{Lottery scheduling}

The basic idea of this kind of scheduling is that the CPU gives the processes lottery tickets for provision of CPU time. A lottery ticket is chosen at random at the time of scheduling decision and the process holding the ticket gets the CPU.[1][2][8]

\section{RELATED WORKS}

In [1], the investigators talked about an algorithm named $A N$ algorithm based on a new methodology, dynamic -time quantum. Its aim is to make the operating system adjust the time quantum so that it goes in pace with the burst time of the set of waiting processes in ready queue. According to this work, they were able to solve fixed time quantum problem, hence increasing the performance of the round robin. The novelists of [2] proposed priority based round robin CPU scheduling algorithm based on the combination of round robin and priority scheduling algorithm. The improved algorithm retained the benefits of round robin in dropping starvation and also integrated the advantages of priority scheduling. The investigators of [3] suggested the modified round robin scheme (MRR) which involved fewer number of arbitration steps in each repetition when compared to iSLIP algorithm. The MRR involved less amount of processing steps. In [4], the novelists did not aim to change the round robin notion but the proposed algorithm worked on reducing the average turnaround time, average waiting time and the number of context switches so as to improve the scheduling of an execution process. The authors in [5] suggested an algorithm that calculated the time quantum in ascending order and sum of the maximum and minimum CPU burst time and multiply 
the result by 80 percentages. In [6], the researchers, recommended an algorithm namely, fuzzy round robin algorithm that tried to remove the problem with the CPU round robin scheduling that is, if the time requisite for the running process is some extent more than time quantum even by a fraction value, then the process gets forestalled and context switch occurs. This algorithm also eliminated the problem with the time quantum of RR scheduling, that is if it is too large, the algorithm degenerates to FCFS or it worsens the performance otherwise. The authors in [7], made slight changes to the conventional round robin algorithm where the time quantum of the scheduled processes is augmented to some amount whose remaining time in its last turn is less than or equal to a given threshold value, which is one-fourth of the time quantum. In [8], the novelists talked about dynamic quantum precision wherein a processor is said to be optimally implemented if it has highest throughput, low wait time, low turnaround time and less no of context switches for process coming to execution. This proposal worked by finding the left out B.T. of processes in the last but one turn of each processed to get an optimal threshold value also the processes has been divided into two categories, first category modifies time quantum and second will be processed as per classical RR algorithm. The researchers of [9] followed a mathematical approach which attempts to mathematically formularize the computation of waiting time of any process in a static $n$ processes, CPU bound a round robin scheme which also calculated other performance measures as well. The approach followed uses two ready queue, wherein a process is returned to second ready queue after completion of last round. This reduces average waiting time and increases throughput while level of C.P.U utilization is preserved and there is no substantial increase in overheads.

\section{PROPOSED APPROACH}

For each process, the square root of the burst time is inferred. In the proposed approach the time quantum is modified for the process which requires a fractional more time than allocated time quantum cycle(s) using the threshold value ' $\mathrm{K}$ ' which is calculated as

$\mathrm{K}=\left\lfloor\left(\mathrm{TQ}-\sum \mathrm{SQ} / \mathrm{N}\right)\right\rfloor$

$\mathrm{SQ}=\operatorname{sqrt}\left(\mathrm{BT}\left[\mathrm{P}_{\mathrm{i}}\right]\right)$

The other parameters are calculated as follows

NOC $[\mathrm{Pi}]=\lceil(\mathrm{BT}[\mathrm{Pi}] / \mathrm{TQ})\rceil$

$\mathrm{RT}[\mathrm{Pi}]=\mathrm{BT}[\mathrm{Pi}] \% \mathrm{TQ}$

The pseudo code given below presents the proposed approach ImpRoundRobin(TQ, BT[i]) \{

assign the $\mathrm{CPU}$ to process $\mathrm{P}[\mathrm{i}]$

$$
\begin{aligned}
& \text { count }[\mathrm{Pi}]=1 ; \\
& \text { ITQ = TQ; } \\
& \text { if }(\mathrm{BT}[\mathrm{i}]<=\mathrm{TQ})
\end{aligned}
$$

else

if $(\mathrm{BT}[\mathrm{Pi}] \% \mathrm{TQ}==0)$

\{

assign $\mathrm{CPU}$ to the process $\mathrm{P}[\mathrm{i}]$ and do

$$
\mathrm{BT}[\mathrm{Pi}]=\mathrm{BT}[\mathrm{Pi}]-\mathrm{TQ} \text {; }
$$$$
\text { count[Pi]++; }
$$

\}

$$
\begin{aligned}
& \text { else if }(\mathrm{BT}[\mathrm{Pi}] \% \mathrm{TQ} !=0) \\
& \{\quad \text { else if }(\mathrm{RT}<=\mathrm{K}) \\
& \text { if }(\mathrm{K}<=\mathrm{TQ} / 2) \\
& \{\quad \mathrm{TQ}=\mathrm{TQ}+\mathrm{K} ;\}\}
\end{aligned}
$$

$$
\begin{aligned}
& \text { if }(\mathrm{K}>\mathrm{TQ} / 2) \\
& \{\quad \mathrm{K}=\mathrm{KTQ} / 2 ;
\end{aligned}
$$

Assign CPU to process $\mathrm{p}[\mathrm{i}]$ and do

$$
\mathrm{TQ}=\mathrm{TQ}+\mathrm{K} ;\}\}
$$

If $\left(\right.$ Count $\left.\left[\mathrm{P}_{\mathrm{i}}\right]==\operatorname{NOC}\left[\mathrm{P}_{\mathrm{i}}\right]-1\right)$

$$
\begin{aligned}
& \{ \\
& \text { NOC[Pi] }-1 \\
& \text { BT=BT-TQ } \\
& \text { TQ=ITQ } \\
& \} \\
& \text { else } \\
& \{
\end{aligned}
$$

Assign CPU to [Pi] and do

$$
\begin{aligned}
& \text { BT[Pi]=BT[Pi]-TQ; } \\
& \text { count [Pi]++; } \\
& \text { TQ=ITQ; } \\
& \} \\
& \text { else } \\
& \{ \\
& \text { BT[Pi]=BT[Pi]-TQ; } \\
& \}\}
\end{aligned}
$$

Table 1(a): Table of processes for example 1 using conventional round robin

\begin{tabular}{|c|c|}
\hline Process Name & Burst Time (BT) \\
\hline P1 & 15 \\
\hline P2 & 16 \\
\hline P3 & 25 \\
\hline P4 & 18 \\
\hline P5 & 31 \\
\hline
\end{tabular}

TIME QUANTUM (TQ): 5

Average Turnaround Time $($ TAT $)=(55+76+94+84+$ $105) / 5=82$

No. of Context Switches $=$ No. of Blocks -1

$=>23-1=22$

Average WT $[\mathrm{Pi}]=\mathrm{TAT}[\mathrm{Pi}]-\mathrm{BT}[\mathrm{Pi}]$

$$
\begin{aligned}
& \Rightarrow(55-15)+(76-16)+(94-25)+(84-18)+(105-31 \\
& \Rightarrow 40+60+69+66+74 \\
& \Rightarrow 309
\end{aligned}
$$

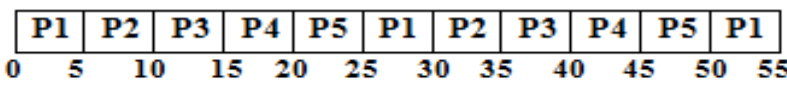

\begin{tabular}{|l|l|l|l|l|l|l|l|l|l|l|l|}
\hline P2 & P3 & P4 & P5 & P2 & P3 & P4 & P5 & P3 & P5 & P5 & P5 \\
\hline 55 & 60 & 65 & 70 & 75 & 76 & $\mathbf{8 1}$ & $\mathbf{8 4}$ & $\mathbf{8 9}$ & $\mathbf{9 4}$ & $\mathbf{9 9}$ & 104105 \\
\hline
\end{tabular}

Figure 1(a): Gantt chart for example 1

Table 1(b): Table of processes for example 1 using proposed approach

\begin{tabular}{|c|c|c|c|c|}
\hline $\begin{array}{c}\text { Process } \\
\text { Name }\end{array}$ & $\begin{array}{c}\text { Burst } \\
\text { Time } \\
(\text { BT) }\end{array}$ & $\begin{array}{c}\text { Square } \\
\text { Root of } \\
\text { the } \\
\text { Burst } \\
\text { Time } \\
\text { (Sq.) }\end{array}$ & $\begin{array}{c}\text { Number } \\
\text { of cycles } \\
\text { (NOC) }= \\
\text { BT / TQ }\end{array}$ & $\begin{array}{c}\text { Remaining } \\
\text { Time (RT) } \\
=\text { BT \% } \\
\text { TQ }\end{array}$ \\
\hline P1 & 15 & 3 & 3 & 0 \\
\hline P2 & 16 & 4 & 3 & 1 \\
\hline P3 & 25 & 5 & 5 & 0 \\
\hline P4 & 18 & 4 & 3 & 3 \\
\hline P5 & 31 & 5 & 6 & 1 \\
\hline
\end{tabular}


TIME QUANTUM (TQ): 5

Number of Processes $(\mathrm{N})=5$

$$
\begin{aligned}
\mathrm{K} & =\left\lfloor\left(\mathrm{TQ}-\sum \mathrm{SQ} / \mathrm{N}\right)\right\rfloor \\
& =5-4 \\
& =1
\end{aligned}
$$

Average Turnaround Time $($ TAT $)=(55+61+94+84+$ $105) / 5=79$

No. of Context Switches $=$ No. of Blocks -1

$\Rightarrow 20-1=19$

Average WT $[\mathrm{Pi}]=\mathrm{TAT}[\mathrm{Pi}]-\mathrm{BT}[\mathrm{Pi}]$

$\Rightarrow(55-15)+(61-16)+(94-25)+(84-18)+(105-31$

\begin{tabular}{|c|c|c|c|c|c|c|c|c|c|}
\hline P1 & P2 & P3 & P4 & P5 & P3 & P4 & P5 & P3 & P5 \\
\hline
\end{tabular}

$=>40+45+69+66+74=>294$

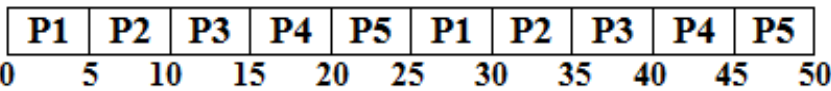

Table 2(a): Table of processes for example 2 using conventional round robin

\begin{tabular}{|c|c|}
\hline $\begin{array}{c}\text { Process } \\
\text { Name }\end{array}$ & $\begin{array}{c}\text { Burst Time } \\
\text { (BT) }\end{array}$ \\
\hline P1 & 31 \\
\hline P2 & 17 \\
\hline P3 & 47 \\
\hline P4 & 48 \\
\hline P5 & 6 \\
\hline
\end{tabular}

TIME QUANTUM (TQ): 15

Average Turnaround Time $($ TAT $)=(114+83+146+149+$ $66) / 5=111$

No. Of context switches $=$ no of blocks -1

$\Rightarrow 14-1=13$

Average WT $[\mathrm{Pi}]=\mathrm{TAT}[\mathrm{Pi}]-\mathrm{BT}[\mathrm{Pi}]$

\begin{tabular}{|c|c|c|c|c|c|c|c|c|c|}
\hline P1 & P2 & P3 & P4 & P5 & P1 & P2 & P3 & P3 & P1 \\
\hline
\end{tabular}

$\Rightarrow(114-31)+(83-17)+(146-47)+(149-48)+(66-6)$

$\Rightarrow 83+66+99+101+60 \Rightarrow 409$

\section{\begin{tabular}{|l|l|l|l|}
\hline P3 & P4 & P3 & P4 \\
\hline
\end{tabular} \\ $\begin{array}{llllll}114 & 129 & 144 & 146 & 149\end{array}$}

Figure 2(a): Gantt chart for example 2:

Table 2(b): Table of processes for example 2 using proposed approach

\begin{tabular}{|c|c|c|c|c|}
\hline $\begin{array}{c}\text { Process } \\
\text { Name }\end{array}$ & $\begin{array}{c}\text { Burst } \\
\text { Time } \\
\text { (BT) }\end{array}$ & $\begin{array}{c}\text { Square } \\
\text { Root of } \\
\text { Burst } \\
\text { time } \\
\text { (Sq.) }\end{array}$ & $\begin{array}{c}\text { Number } \\
\text { of } \\
\text { cycles } \\
\text { (NOC) } \\
=\text { BT / } \\
\text { TQ }\end{array}$ & $\begin{array}{c}\text { Remaining } \\
\text { Time (RT) } \\
\text { = BT } \\
\text { \% TQ }\end{array}$ \\
\hline P1 & 31 & 5 & 2 & 1 \\
\hline P2 & 17 & 4 & 1 & 2 \\
\hline P3 & 47 & 6 & 3 & 2 \\
\hline P4 & 48 & 6 & 3 & 3 \\
\hline P5 & 6 & 2 & 0 & 0 \\
\hline
\end{tabular}

TIME QUANTUM (TQ): 15

Number of process $(\mathrm{N})=5$

$$
\begin{aligned}
\mathrm{K} & =\left\lfloor\left(\mathrm{TQ}-\sum \mathrm{SQ} / \mathrm{N}\right)\right\rfloor \\
& =15-4 \\
& =11
\end{aligned}
$$

Average Turnaround Time $($ TAT $)=(84+32+131+$ $114+149) / 5=102$

No. Of context switches $=$ no of blocks -1

$\Rightarrow 10-1=9$

Average WT $[\mathrm{Pi}]=\mathrm{TAT}[\mathrm{Pi}]-\mathrm{BT}[\mathrm{Pi}]$

$\Rightarrow(84-31)+(32-17)+(131-47)+(114-48)+(149-6)$

$=>53+15+84+66+143=361$

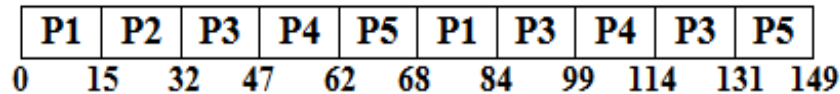

Figure 2(b): Gantt chart for example 2:

Table 3(a): Table of processes for example 3 using conventional round robin

\begin{tabular}{|c|c|}
\hline Process Name & Burst Time \\
\hline P1 & 12 \\
\hline P2 & 23 \\
\hline P3 & 31 \\
\hline P4 & 41 \\
\hline P5 & 32 \\
\hline
\end{tabular}

TIME QUANTUM (TQ): 10

Average Turnaround Time $($ TAT $)=(52+95+126+139+$ 138)/ $5=110$

No. Of context switches $=$ no of blocks -1

\begin{tabular}{|c|c|c|c|c|c|c|c|c|c|}
\hline P1 & P2 & P3 & P4 & P5 & P1 & P2 & P3 & P4 & P5 \\
\hline
\end{tabular}

$\Rightarrow 18-1=17$

\begin{tabular}{|c|c|c|c|c|c|c|c|}
\hline P2 & P3 & P4 & P5 & \begin{tabular}{|l|l} 
P3 \\
\end{tabular} & P4 & P5 & P4 \\
\hline
\end{tabular}

Average WT $[\mathrm{Pi}]=\mathrm{TAT}[\mathrm{Pi}]-\mathrm{BT}[\mathrm{Pi}]$

$\Rightarrow(52-12)+(95-23)+(126-31)+(139-41)+(138-$

32)

$=>40+72+95+98+106=411$

Figure 3(a): Gantt chart for example 3:

Table 3(b): Table of processes for example 3 using proposed approach

\begin{tabular}{|c|c|c|c|c|}
\hline $\begin{array}{c}\text { Process } \\
\text { Name }\end{array}$ & $\begin{array}{c}\text { Burst } \\
\text { Time } \\
\text { (BT) }\end{array}$ & $\begin{array}{c}\text { Square } \\
\text { root of } \\
\text { the } \\
\text { Burst } \\
\text { Time } \\
\text { (Sq) }\end{array}$ & $\begin{array}{c}\text { Number } \\
\text { of } \\
\text { cycles } \\
\text { (NOC) } \\
=\mathbf{B T} / \\
\text { TQ }\end{array}$ & $\begin{array}{c}\text { Remaining } \\
\text { Time (RT) } \\
=\mathbf{B T} \% \\
\text { TQ }\end{array}$ \\
\hline P1 & 12 & 3 & 1 & 2 \\
\hline P2 & 23 & 4 & 2 & 3 \\
\hline P3 & 31 & 5 & 3 & 1 \\
\hline P4 & 41 & 6 & 4 & 1 \\
\hline P5 & 32 & 5 & 3 & 2 \\
\hline
\end{tabular}

TIME QUANTUM (TQ): 10

Number of process $(\mathrm{N})=5$ 


$$
\begin{aligned}
\mathrm{K} & =\left\lfloor\left(\mathrm{TQ}-\sum_{\mathrm{SQ}} / \mathrm{N}\right)\right\rfloor \\
& =10-4=6
\end{aligned}
$$

Average Turnaround Time $($ TAT $)=(12+65+106+139+$ 128) $/ 5=90$

No. Of context switches $=$ no of blocks -1

$\Rightarrow 13-1=12$

Average WT $[\mathrm{Pi}]=$ TAT $[\mathrm{Pi}]-\mathrm{BT}[\mathrm{Pi}]$

$\Rightarrow(12-12)+(65-23)+(106-31)+(139-41)+(128-$

32)

\begin{tabular}{|c|c|c|c|c|c|c|c|c|c|}
\hline P1 & P2 & P3 & P4 & P5 & P2 & P3 & P4 & P5 & P3 \\
\hline
\end{tabular}

$=>0+42+75+98+96=311$

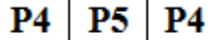

$\begin{array}{llll}106 & 116 & 128 & 139\end{array}$

Figure 3(b): Gantt chart for example 3:

Table 4(a): Table of processes for example 4 using conventional round robin

\begin{tabular}{|c|c|}
\hline Process Name & Burst Time \\
\hline P1 & 31 \\
\hline P2 & 17 \\
\hline P3 & 47 \\
\hline P4 & 48 \\
\hline P5 & 16 \\
\hline
\end{tabular}

TIME QUANTUM: 15

Average Turnaround Time $($ TAT $)=(124+92+156+159+$ $123) / 5=130$

No. Of context switches $=$ no of blocks -1

$\Rightarrow 15-1=14$

Average WT $[\mathrm{Pi}]=\mathrm{TAT}[\mathrm{Pi}]-\mathrm{BT}[\mathrm{Pi}]$

$\Rightarrow(124-31)+(92-15)+(156-47)+(159-48)+(123-$

16)

$=>93+77+109+111+107=497$

\begin{tabular}{|c|c|c|c|c|c|c|c|c|c|c|}
\hline P1 & P2 & P3 & P4 & P5 & P1 & P2 & P3 & P4 & P5 \\
\hline 0 & 15 & 30 & 45 & 60 & 75 & 90 & 92 & 107 & 122 & 11
\end{tabular}

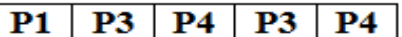

$\begin{array}{llllll}123 & 124 & 139 & 154 & 156 & 159\end{array}$

Figure 4(a): Gantt chart for example 4:

Table 4(b): Table of processes for example 4 using proposed approach

\begin{tabular}{|c|c|c|c|c|}
\hline $\begin{array}{c}\text { Process } \\
\text { Name }\end{array}$ & $\begin{array}{c}\text { Burst } \\
\text { Time } \\
\text { (BT) }\end{array}$ & $\begin{array}{c}\text { Square } \\
\text { root of } \\
\text { the } \\
\text { burst } \\
\text { time } \\
\text { (Sq.) }\end{array}$ & $\begin{array}{c}\text { Number } \\
\text { of } \\
\text { cycles } \\
\text { (NOC) } \\
=\mathbf{B T} / \\
\text { TQ }\end{array}$ & $\begin{array}{c}\text { Remaining } \\
\text { Time (RT) } \\
=\mathbf{B T} \text { \% } \\
\text { TQ }\end{array}$ \\
\hline P1 & 31 & 5 & 2 & 1 \\
\hline P2 & 17 & 4 & 1 & 2 \\
\hline P3 & 47 & 6 & 3 & 2 \\
\hline P4 & 48 & 6 & 3 & 3 \\
\hline P5 & 16 & 4 & 4 & 1 \\
\hline
\end{tabular}

TIME QUANTUM: 15

$$
\begin{aligned}
\mathrm{K} & =\left\lfloor\left(\mathrm{TQ}-\sum \mathrm{SQ} / \mathrm{N}\right)\right\rfloor \\
& =15-5 \\
& =10
\end{aligned}
$$

Average Turnaround Time $($ TAT $)=(94+32+141+159+$ $78) / 5=100$

No. Of context switches $=$ no of blocks -1

$\Rightarrow 10-1=9$

Average WT $[\mathrm{Pi}]=\mathrm{TAT}[\mathrm{Pi}]-\mathrm{BT}[\mathrm{Pi}]$

$\Rightarrow(94-31)+(32-15)+(141-47)+(159-48)+(78-$

16)

$=>69+17+94+111+62=345$

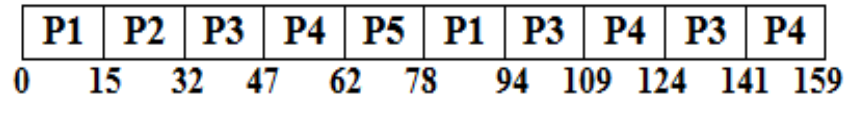

Figure 4(b): Gantt chart for example 4:

\section{RESULTS}

The table and figures presented below shows the comparison between the proposed approach and the conventional round robin Algorithm.

Table 5: Comparison table for example 1

\begin{tabular}{|l|l|l|l|}
\hline & $\begin{array}{l}\text { Average } \\
\text { Turnaround } \\
\text { Time (TAT) }\end{array}$ & $\begin{array}{l}\text { Average } \\
\text { Wait } \\
\text { Time }\end{array}$ & $\begin{array}{l}\text { No. of } \\
\text { Context } \\
\text { Switches }\end{array}$ \\
\hline $\begin{array}{l}\text { CONVENTIONAL } \\
\text { ROUND ROBIN }\end{array}$ & 82 & 309 & 22 \\
\hline $\begin{array}{l}\text { PROPOSED } \\
\text { APPROACH }\end{array}$ & 79 & 269 & 9 \\
\hline
\end{tabular}

Table 6: Comparison table for example 2

\begin{tabular}{|l|l|l|l|}
\hline & $\begin{array}{l}\text { Average } \\
\text { Turnaround } \\
\text { Time }\end{array}$ & $\begin{array}{l}\text { Average } \\
\text { Wait } \\
\text { Time }\end{array}$ & $\begin{array}{l}\text { No. of } \\
\text { Context } \\
\text { Switches }\end{array}$ \\
\hline $\begin{array}{l}\text { CONVENTIONAL } \\
\text { ROUND ROBIN }\end{array}$ & 111 & 409 & 13 \\
\hline $\begin{array}{l}\text { PROPOSED } \\
\text { APPROACH }\end{array}$ & 102 & 361 & 9 \\
\hline
\end{tabular}

Table 7: Comparison table for example 3

\begin{tabular}{|l|l|l|l|}
\hline & $\begin{array}{l}\text { Average } \\
\text { Turnaround } \\
\text { Time }\end{array}$ & $\begin{array}{l}\text { Average } \\
\text { Wait } \\
\text { Time }\end{array}$ & $\begin{array}{l}\text { No. of } \\
\text { Context } \\
\text { Switches }\end{array}$ \\
\hline $\begin{array}{l}\text { CONVENTIONAL } \\
\text { ROUND ROBIN }\end{array}$ & 110 & 411 & 17 \\
\hline $\begin{array}{l}\text { PROPOSED } \\
\text { APPROACH }\end{array}$ & 90 & 311 & 12 \\
\hline
\end{tabular}


Table 8: Comparison table for example 4

\begin{tabular}{|l|l|l|l|}
\hline & $\begin{array}{l}\text { Average } \\
\text { Turnaround } \\
\text { Time }\end{array}$ & $\begin{array}{l}\text { Average } \\
\text { Wait } \\
\text { Time }\end{array}$ & $\begin{array}{l}\text { No. of } \\
\text { Context } \\
\text { Switches }\end{array}$ \\
\hline $\begin{array}{l}\text { CONVENTIONAL } \\
\text { ROUND ROBIN }\end{array}$ & 130 & 497 & 14 \\
\hline $\begin{array}{l}\text { PROPOSED } \\
\text { APPROACH }\end{array}$ & 69 & 347 & 9 \\
\hline
\end{tabular}

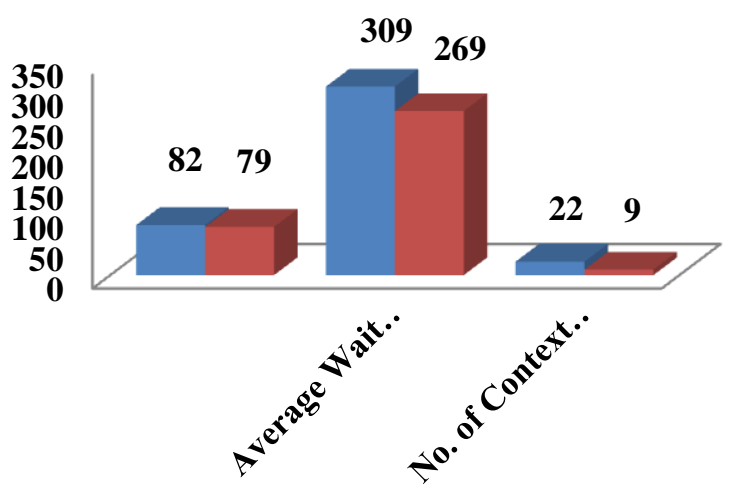

\section{CONVENTIONAL ROUND ROBIN} $\square$ PROPOSED APPROACH

Figure 5: comparison graph for example 1

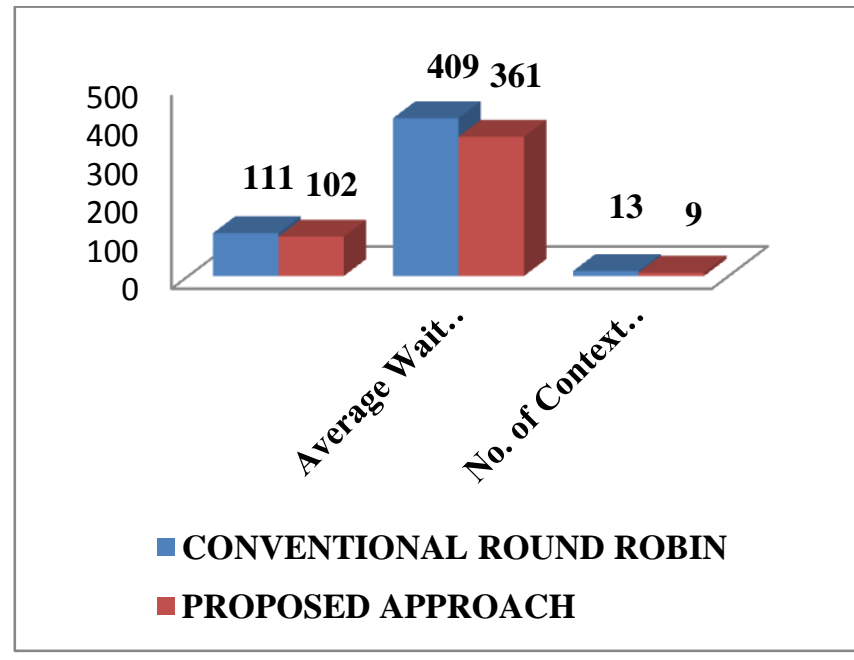

Figure 6: comparison graph for example 2

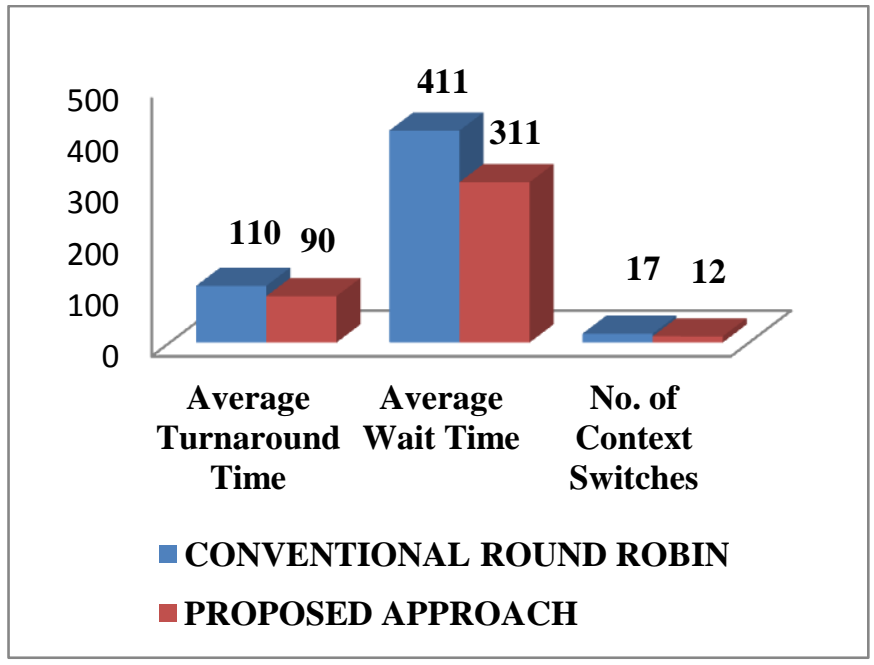

Figure 7: comparison graph for example 3

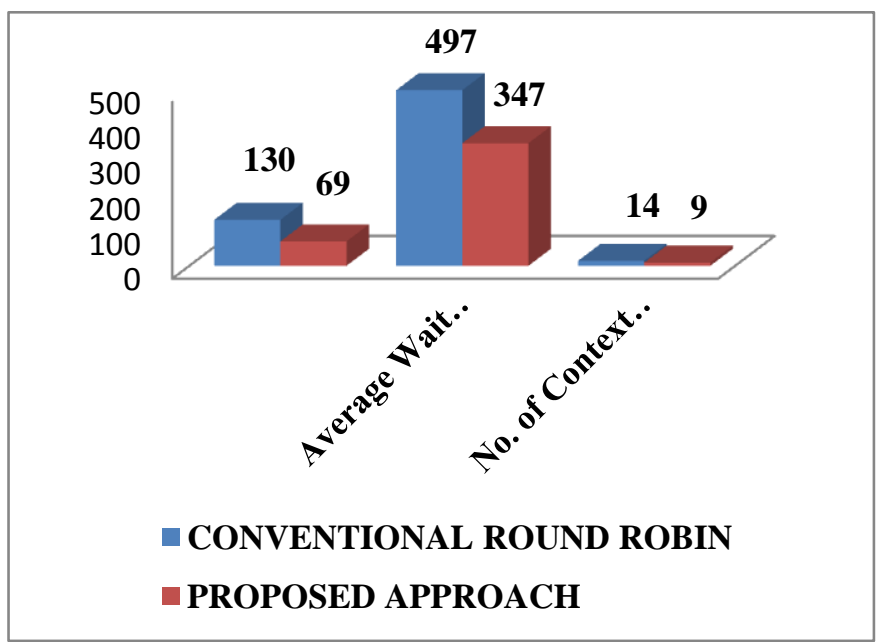

Figure 4: comparison graph for example 4

\section{CONCLUSION}

It can be observed that the proposed approach has a clear edge of over the conventional round robin algorithm. The similar approach can be applied to Real Time operating systems environment with only slight modifications.

\section{REFERENCES}

[1] Abbas Noon, Ali Kalakech, and Seifedine Kadry, A New Round Robin Based Scheduling Algorithm for Operating Systems: Dynamic Quantum Using the Mean Average, IJCSI International Journal of Computer Science Issues, Vol. 8, Issue 3, No. 1, pp. 224-229, May 2011.

[2] Haidar M. Ali and Kaies Khalid, An Improvement on Round Robin Scheduling Method, ICITNS 2003 International Conference on Information Technology and Natural Sciences.

[3] Shanmugam Arumugam and Shanthi Govindaswamy, Performance of the Modified Round Robin Scheduling Algorithm for Input Queued Switches Under SelfSimilar Traffic, The International Arab Journal of Information Technology, Vol. 3, No 2, pp. 165-171 ,April 2006. 
[4] KN Rout, G. Das, B.M. Sahoo, A.K. Agrawalla, ,Improving Average Waiting Time Using Dyanamic Time Quantum,ISSN (Print): 2319-2526, Volume - I, Issue-2.2012.

[5] Ali Jbaeer Dawood, Improving Efficiency of Round Robin using Ascending Quantum and MinimumMaximum Burst Time- Journal of University of Anbar for pure science: Vol.6:NO.2,2012.

[6] Bashir Alam, Fuzzy Round Robin C.P.U Scheduling Algorithm , Journal of Computer Science , pp. 10791085,2013

[7] Mohd Abdul Ahad, Modifying Round Robin Algorithm for Process Scheduling using Dynamic Quantum Precision, IJCA Special Issue on Issues and Challenges in Networking, Intelligence and Computing Technologies ICNICT, pp. 5-10, November.2012.
[8] D. Pandey and Vandana, Improved Round Robin Policy A Mathematical Approach, D. Pandey et. al. / (IJCSE) International Journal on Computer Science and Engineering, Vol. 02, No. 04, pp. 948-954, 2010.

[9] Lalit Kishor , Dinesh Goyal, Time Quantum Based Improved Scheduling Algorithm, Volume3, Issue International Journal of Advanced Research in Computer Science and Software Engineering. ISSN: 2277 128X, 4, April, 2013.

[10] Silberschatz ,Galvin and Gagne, Operating systems concepts, 8th edition, Wiley, 2009.

[11] D.M. Dhamdhare, Operating Systems, Tata McGraw Hill, Second Edition,2006. 